\title{
Effect of CPU-XT-008, a combretastatin A-4 analogue, on the proliferation, apoptosis and expression of vascular endothelial growth factor and basic fibroblast growth factor in human umbilical vein endothelial cells
}

\author{
RUI XIONG ${ }^{1}$, JING SUN $^{1}, \mathrm{KUN} \mathrm{LIU}^{2}, \mathrm{YUNGEN} \mathrm{XU}^{2}$ and SHUYING HE ${ }^{1}$ \\ ${ }^{1}$ School of Life Science and Technology, China Pharmaceutical University, Nanjing, Jiangsu 211198; \\ ${ }^{2}$ School of Pharmacy, China Pharmaceutical University, Nanjing, Jiangsu 210009, P.R. China
}

Received December 14, 2014; Accepted September 22, 2015

DOI: $10.3892 / \mathrm{ol} .2015 .3867$

\begin{abstract}
The present study investigated the effect of the combretastatin A-4 analogue CPU-XT-008 on the proliferation, apoptosis and expression of vascular endothelial growth factor (VEGF) and basic fibroblast growth factor (FGF-2) in human umbilical vein endothelial cells (HUVECs). The proliferation capacity of HUVECs was analyzed with a cell viability assay, while their apoptosis and migration abilities were evaluated via flow cytometry and monolayer denudation assay, respectively. The mRNA and protein expression levels of VEGF and FGF-2 in these cells were determined by reverse transcription-polymerase chain reaction, and cell-based ELISA, western blotting and immunocytochemistry, respectively. The results demonstrated that CPU-XT-008 inhibited proliferation and migration, and induced apoptosis in HUVECs in a dose-dependent manner. In addition, CPU-XT-008 downregulated the mRNA and protein expression levels of VEGF and FGF-2 in these cells. These findings suggest that CPU-XT-008 exerts anti-angiogenic effects in HUVECs, which may explain the inhibition of cell proliferation and migration, induction of apoptosis, and reduction in the mRNA and protein expression levels of VEGF and FGF-2 observed in the present study.
\end{abstract}

\section{Introduction}

Cancer, one of the leading causes of mortality in the world, is induced by a plethora of external and internal factors (1). Accordingly, various types of therapeutic strategies against cancer have been investigated (2). One of these therapeutic

Correspondence to: Professor Shuying He, School of Life Science and Technology, China Pharmaceutical University, 24 Tongjia Road, Nanjing, Jiangsu 211198, P.R. China

E-mail: heshuying92@126.com

Key words: combretastatin A-4, human umbilical vein endothelial cells, CPU-XT-008, vascular endothelial growth factor, basic fibroblast growth factor strategies involves the use of vascular disrupting agents, the action of which may be due to their ability to prevent the formation of blood vessels (anti-angiogenic agents) or to promote the destruction of existing blood vessels (anti-vascular agents) (3).

A functional network of blood vessels is crucial for the growth, development and metastasis of solid tumors (4). While small tumors $(<2 \mathrm{~mm})$ receive oxygen and nutrients from the surrounding tissue by diffusion processes, larger tumors must develop an angiogenic phenotype to ensure their own supply of oxygen and nutrients (5). Tumor angiogenesis involves several steps, including proliferation of endothelial cells, proteolysis of the extracellular matrix, and migration of endothelial cells, which lead to the formation of tumor blood vessels (4). Endothelial cells are desirable targets for cancer therapies due to their accessibility and genetic stability. In addition, unlike tumor cells, endothelial cells do not readily acquire drug resistance $(6,7)$. Therefore, new born calf serum (NBCS)-induced human umbilical vein endothelial cells (HUVECs) were selected as tumor cell model in the present study.

Tumor angiogenesis is a complex process, and involves the tight interplay of numerous factors (8). Vascular endothelial growth factor (VEGF) and basic fibroblast growth factor (FGF-2) are key regulators of angiogenesis, since they promote the survival, proliferation and migration of endothelial cells, while increasing vascular permeability (9). Overexpression of VEGF and FGF-2 has been reported to occur in various types of tumors (10). Thus, VEGF and FGF-2 have become key target molecules in cancer therapy (11).

Combretastatin A-4 (CA-4) is as an effective anti-vascular agent that was originally isolated in the late 1980s from the bark of the African bush willow tree Combretum caffrum (12). In our unpublished preliminary studies, we aimed to overcome the poor water solubility and bioavailability of CA-4, the amino sugar structure 1-glucosamine was coupled with CA-4, which resulted in a series of novel CA-4 derivatives containing an amino sugar. These CA-4 derivatives were capable of simulating the function of heparin, which is used to regulate vascular factors such as VEGF and FGF-2 (13). In addition, the aforementioned CA-4 derivatives also displayed molecular recognition and biocompatibility properties (14). Among these novel CA-4 derivatives, the compound termed 
CPU-XT-008 was identified to be the most active of the series, in our preliminary studies. Thus, the present study hypothesized that CPU-XT-008 may be able to inhibit the proliferation and migration of HUVECs, and stimulate apoptosis in these cells. The effects of CPU-XT-008 on the messenger (m)RNA and protein levels of VEGF and FGF-2 were examined in the present study. The results obtained may aid to determining the exact anti-vascular mechanism of CPU-XT-008.

\section{Materials and methods}

Reagents and cell culture. CPU-XT-008 was provided by the Medicinal Chemistry Department of China Pharmaceutical University (Nanjing, China). The compound was dissolved in dimethylsulfoxide (DMSO) at a concentration of $10 \mathrm{mmol} / \mathrm{l}$, stored at $-20^{\circ} \mathrm{C}$, and diluted in Dulbecco's modified Eagle's medium (DMEM) (Gibco, Thermo Fisher Scientific, Inc., Waltham, MA, USA) for cell culture experiments. HUVECs were kindly provided by the Biochemistry and Microbiology Laboratory of China Pharmaceutical University. The adherent cells were maintained in DMEM supplemented with $10 \%$ NBCS (Gibco, Thermo Fisher Scientific, Inc.) in a humidified incubator at $37^{\circ} \mathrm{C}$ with $5 \% \mathrm{CO}_{2}$. HUVECs that had undergone four-eight passages were grown to confluence, detached by trypsin (Sigma-Aldrich, St. Louis, MO, USA), suspended at an appropriate concentration in culture medium, and seeded into culture dishes or well plates for subsequent experimental use.

Methyl thiazolyl tetrazolium (MTT) was purchased from Sigma-Aldrich. VEGF rabbit anti-human IgG (cat no. PB0084), FGF-2 rabbit anti-human IgG (cat no. BA14189), $\beta$-actin rabbit anti-human IgG (cat no. BA2305), and hoseradish peroxidase-conjugated goat anti-rabbit IgG (cat no. BA1055) were obtained from Boster Systems, Inc. (Wuhan, China). 3,3',5,5'-tetramethylbenzidine (TMB) was obtained from Beyotime Institute of Biotechnology (Haimen, China). RevertAid First Strand cDNA Synthesis Kit was acquired from Fermentas, Thermo Fisher Scientific, Inc. The cell apoptosis detection kit used in the present study was purchased from Beckman Coulter, Inc. (Brea, CA, USA), while ECL Advance Detection Kit, the Immunohistochemistry detection system kit (cat no. BD5100) and DAB Chromogen Kit (cat no. BD5038) were obtained from Bioworld Technology, Inc. (St. Louis Park, MN, USA). All other chemicals were of analytical grade.

Cell proliferation assay. The growth of HUVECs was determined by the ability of the living cells to reduce the yellow MTT dye to a purple formazan product. For the assay, HUVECs were detached, seeded at a concentration of $2 \times 10^{4}$ cells/well in 96-well plates, and allowed to adhere overnight. CPU-XT-008 was then added to each well, and the cells were incubated for additional $24 \mathrm{~h}$. Next, $20 \mu \mathrm{l}$ MTT (5 mg/ml) was added to each well, and $4 \mathrm{~h}$ later the formazan product was dissolved in $150 \mu \mathrm{l}$ DMSO. The absorbance of the solution was subsequently measured by iMark ${ }^{\mathrm{TM}}$ Microplate Absorbance Reader (Bio-Rad, Hercules, CA, USA). Cell growth was expressed as the absorbance of the reduced dye at $570 \mathrm{~nm}$. Each assay was performed $\geq$ three times, using a matrix of 12 replicate wells per each concentration of drug tested.
Apoptosis analysis. Cell death was assessed by flow cytometry at $24 \mathrm{~h}$ post-treatment with CPU-XT-008. Cells floating in the supernatant and adherent cells harvested by trypsin were combined to create a single cell suspension. The cells were then pelleted by centrifugation, and washed with phosphate-buffered saline (PBS). Next, $2 \times 10^{6}$ cells were pelleted and suspended in $200 \mu \mathrm{l}$ buffer solution, followed by staining with annexin V-fluorescein isothiocyanate and propidium iodide (PI) (BD Biosciences, Franklin Lakes, NJ, USA), according to the manufacturer's protocol. The cells were then diluted with $300 \mu \mathrm{l}$ buffer solution, and processed for data acquisition and analysis on a FACSCalibur flow cytometer (BD Biosciences), using CellQuest Pro software (BD Biosciences). The $\mathrm{X}$ and Y-axes corresponding to the graphical representation of the data indicated the fluorescence intensity associated with the binding of annexin V and PI to the cells, respectively. The percentages of gated populations in the four regions delineated in the graph were detected and subjected to quantitative comparison. Thus, three cell groups were identified by flow cytometry: i) Normal control cells, which were not stained with annexin $\mathrm{V}$ and PI (annexin $\mathrm{V}^{-} / \mathrm{PI}^{-}$); ii) cells in the early stages of apoptosis, which were stained with annexin $\mathrm{V}$ but not with PI (annexin $\mathrm{V}^{+} / \mathrm{PI}^{-}$); and iii) cells in the late stages of apoptosis or necrotic cells, which were stained with annexin $\mathrm{V}$ and PI (annexin $\mathrm{V}^{+} / \mathrm{PI}^{+}$).

Cell wounding. A monolayer denudation assay was used to study cell migration. For the experiment, HUVECs were grown to confluency in 6-well plates, and then starved for $12 \mathrm{~h}$ in serum-free medium in order to achieve cell synchronization. A sterile microtip was then used to create a clean wound through the monolayer, by debriding the cells at an angle of $\sim 30$ degrees. Next, the plates were washed with fresh medium to remove the debrided cells. The adherent cells were then exposed to different concentrations of CPU-XT-008, and allowed to proliferate and migrate in the medium. The wound was assessed at 0 and $24 \mathrm{~h}$ with an Eclipse TS100 microscope (Nikon Corporation, Tokyo, Japan) at 40x magnification, and representative images of three independent experiments were captured. The width of the wound was measured in four random areas, and compared with the initial width.

Cell-based enzyme-linked immunosorbent assay (ELISA). A cell-based ELISA was used to determine the induction time at which the NBCS-induced expression levels of VEGF and FGF-2 were maximum. For the experiment, HUVECs were seeded at a concentration of $2 \times 10^{4}$ cells/well in 96-well plates, and allowed to adhere overnight. Cells were starved for cell synchronization purposes by incubation in serum-free medium for $12 \mathrm{~h}$, followed by incubation in DMEM with $10 \%$ NBCS. Cell-based ELISAs were performed at 0, 2, 4, 6, 8, 10, 12 and $24 \mathrm{~h}$. At the indicated time points, the cells were fixed with $4 \%$ paraformaldehyde $(\mathrm{pH} \mathrm{7.4)}$ ) for $20 \mathrm{~min}$ at room temperature, and then washed 3 times with PBS containing $0.3 \%(\mathrm{v} / \mathrm{v})$ Triton X-100 at room temperature, each time for $5 \mathrm{~min}$. Endogenous peroxidase activity was inactivated with $0.6 \%$ hydrogen peroxide for $20 \mathrm{~min}$ at room temperature. Cells were then washed 3 times for $5 \mathrm{~min}$, as above, and incubated for $1 \mathrm{~h}$ in the aforementioned PBS-Triton X-100 solution, to which $10 \%(\mathrm{w} / \mathrm{v})$ bovine serum albumin (BSA; $1 \mathrm{~g}$ BSA in 
Table I. Primers used for the amplification of VEGF and FGF-2 by reverse transcription-polymerase chain reaction, and sizes of the expected products.

\begin{tabular}{llc} 
Primer & \multicolumn{1}{c}{ Sequence (5'-3') } & Product size (base pairs) \\
\hline VEGF & & 586 and 495 \\
Forward & GGGCCTCCGAAACCATGAAC \\
Reverse & CTGGTTCCCGAAACCCTGAG & \\
FGF-2 & & 237 \\
Forward & GTGTGTGCTAACCGTTACCT & \\
Reverse & CTCTTAGCAGACATTGGAAG & 451 \\
GAPDH & & \\
Forward & ACCACAGTCCATGCCATCAC & \\
Reverse & TCCACCACCCTGTTGCTGTA & \\
\hline
\end{tabular}

VEGF, vascular endothelial growth factor; FGF-2, basic fibroblast growth factor; GAPDH, glyceraldehyde 3-phosphate dehydrogenase.

$10 \mathrm{ml}$ PBS/Triton X-100) was added. Primary monoclonal antibodies against VEGF and FGF-2 were then applied to the cells at a 1:200 dilution, and incubated at $4^{\circ} \mathrm{C}$ overnight. Upon washing, $50 \mu \mathrm{l}$ HRP-conjugated goat anti-rabbit immunoglobulin $\mathrm{G}$ (dilution, 1:1,000) was added to the plates, and incubated for $1 \mathrm{~h}$ at $37^{\circ} \mathrm{C}$. Next, the plates were washed, prior to the addition of TMB. Following 10-15 min incubation at room temperature, the reaction was stopped by adding $50 \mu \mathrm{l}$ $\mathrm{H}_{2} \mathrm{SO}_{4}(2 \mathrm{~mol} / \mathrm{l})$, and the absorbance was subsequently read at $450 \mathrm{~nm}$.

To adjust the results according to the cell density in each well, the cells were stained with crystal violet. For this purpose, the plates were washed and heat-dried, followed by $30-\mathrm{min}$ incubation at room temperature with $100 \mu \mathrm{l}$ crystal violet. The plates were then washed with double distilled water, and $100 \mu \mathrm{l}$ of $1 \%$ sodium dodecyl sulfate (SDS) was added to each well. Following 1-h incubation at room temperature with agitation, the absorbance was read at $595 \mathrm{~nm}$, and the results were expressed by normalizing the values to the cell density $\left(\mathrm{A}_{450} / \mathrm{A}_{595}\right)$.

Western blot analysis. HUVECs were incubated with different concentrations of CPU-XT-008 for $24 \mathrm{~h}$, and subsequently collected, centrifuged, and washed two times with ice-cold PBS. In order to lyse the cells, the cell pellet was then resuspended in lysis buffer containing protease inhibitors $(0.5 \%$ NP-40, $0.25 \%$ sodium deoxycholate, $0.05 \%$ SDS, $150 \mathrm{mM}$ $\mathrm{NaCl}, 50 \mathrm{mM}$ HEPES, $\mathrm{pH} 7.4$ ), and incubated on ice for $30 \mathrm{~min}$. Next, the cell lysates were centrifuged at $12,000 \mathrm{x} \mathrm{rpm}$ at $4^{\circ} \mathrm{C}$ for $10 \mathrm{~min}$, and the supernatants were collected. The protein concentration in the supernatants was determined with BCA Protein Assay Kit (Thermo Fisher Scientific, Inc.). The cell lysates were then mixed with sample buffer (cat no. C516031; dilution 1:4; Sangon Biotech, Shanghai, China), and heated for $5 \mathrm{~min}$ at $95^{\circ} \mathrm{C}$. Proteins $(50 \mu \mathrm{g} / \mathrm{lane})$ were resolved by SDS-polyacrylamide gel electrophoresis in $12 \%$ acrylamide gels, prior to be transferred to polyvinylidene fluoride membranes (Merck Millipore, Darmstadt, Germany). The membranes were blocked with skimmed milk for $2 \mathrm{~h}$ at room temperature, followed by overnight incubation at $4^{\circ} \mathrm{C}$ with rabbit anti-human polyclonal antibodies against VEGF,
FGF-2 and $\beta$-actin (dilution, 1:400). The membranes were then washed 10 min with 3 times in $1 \mathrm{X}$ Tris-buffered saline (TBS; pH 7.4) with $0.05 \%$ Tween-20, and incubated with HRP-conjugated secondary antibodies (dilution, 1:5,000) for $1 \mathrm{~h}$, prior to be visualized with ECL Advance Detection Kit. The protein expression levels of VEGF and FGF-2 were quantified by densitometry, and normalized against the protein expression levels of $\beta$-actin, using NIH ImageJ 1.38 software (National Institutes of Health, Bethesda, MD, USA).

Reverse transcription $(R T)$-polymerase chain reaction $(P C R)$ analysis. HUVECs at 80-90\% confluence were collected following serum starvation for $12 \mathrm{~h}$, and incubated with different concentrations of CPU-XT-008 for $24 \mathrm{~h}$. The cells were then collected, and the total RNA content was isolated from these cells using TRIzol (Thermo Fisher Scientific, Inc.), trichloromethane and isopropanol (Nanjing Chemical Reagent, Nanjing, China), according to the manufacturer's protocol. Overall, $2 \mu 1$ of total RNA $(\sim 1 \mu \mathrm{g} / \mu \mathrm{l})$ was converted to first-strand complementary (c)DNA using RevertAid First Strand cDNA Synthesis Kit. Semi-quantitative PCR was performed in a total volume of $25 \mu \mathrm{l}$ in the presence of Taq Master Mix Loading Dye (Generay Biotech Co., Ltd., Shanghai, China) on a PCR System (Bio-Rad Laboratories, Inc., Hercules, CA, USA). The PCR products were analyzed by electrophoresis on 1.5\% agarose gels (cat no. G-10; Biowest, Kansas City, MO, USA), and visualized under ultraviolet transillumination with Tanon 1600 Gel Imaging System (Tanon Science and Technology Co., Ltd., Shanghai, China). The sequences of the primers used in RT-PCR are listed in Table I. The PCR conditions were as follows: i) For amplification of VEGF, the cycling parameters selected were $94^{\circ} \mathrm{C}$ for $30 \mathrm{sec}, 58^{\circ} \mathrm{C}$ for $1 \mathrm{~min}$, and $72^{\circ} \mathrm{C}$ for $110 \mathrm{sec}$; ii) for FGF-2, the parameters were $94^{\circ} \mathrm{C}$ for $30 \mathrm{sec}, 58^{\circ} \mathrm{C}$ for $50 \mathrm{sec}$, and $72^{\circ} \mathrm{C}$ for $50 \mathrm{sec}$; and iii) for glyceraldehyde 3-phosphate dehydrogenase (GAPDH), the parameters were $94^{\circ} \mathrm{C}$ for $30 \mathrm{sec}, 58^{\circ} \mathrm{C}$ for $50 \mathrm{sec}$, and $72^{\circ} \mathrm{C}$ for $50 \mathrm{sec}$. The expression levels of GAPDH were used to monitor the integrity of the RNA in the samples and to normalize the results, since the quantity of RNA in each sample may vary slightly, depending on the accuracy of loading. 


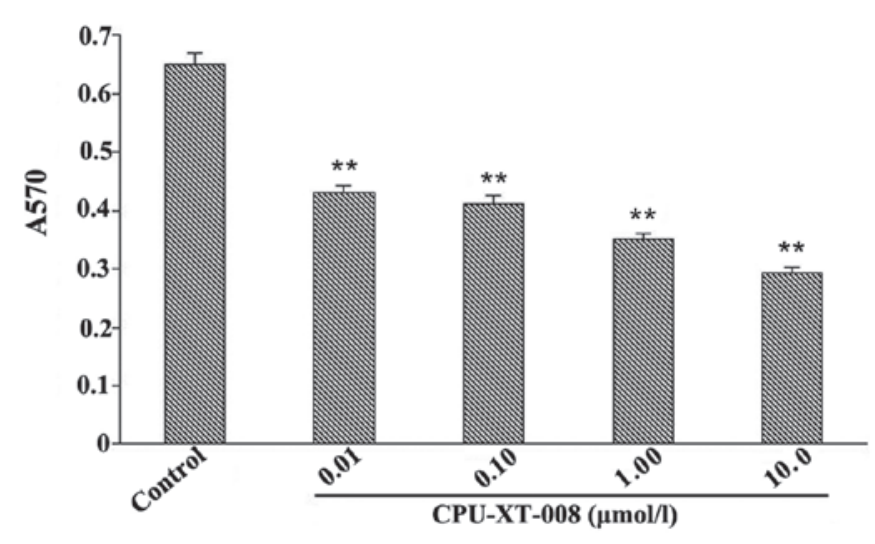

Figure 1. Results of MTT assay, indicating the inhibition of proliferation of human umbilical vein endothelial cells by CPU-XT-008 in a dose-dependent manner. The cells in the control group were treated with DMEM $+10 \%$ NBCS, whereas the cells in the test groups were treated with DMEM $+10 \%$ NBCS + CPU-XT-008 $(0.01,0.10,1.00$ and $10.0 \mu \mathrm{mol} / 1$, respectively). Values are expressed as the mean \pm standard deviation, $n=6 .{ }^{* *} \mathrm{P}<0.01$ vs. Control group. DMEM, Dulbecco's modified Eagle's medium; NBCS, new born calf serum; A, absorbance.

Immunocytochemistry. HUVECs were subjected to immunocytochemical analysis in order to identify the cellular localization of VEGF and FGF-2. In this procedure, cells were grown on coverslips, and fixed with $4 \%$ paraformaldehyde. Endogenous peroxidase activity was inactivated by incubation with $0.6 \%$ hydrogen peroxide (30\% $\mathrm{H} 2 \mathrm{O} 2$ in PBS) for $15 \mathrm{~min}$ at room temperature. Nonspecific binding was blocked by incubation with $10 \%$ BSA in TBS-Tween 20 at $4^{\circ} \mathrm{C}$ for $1 \mathrm{~h}$. Cells were then washed 3 times for $5 \mathrm{~min}$, prior to be incubated overnight at $4^{\circ} \mathrm{C}$ with primary monoclonal antibodies against VEGF and FGF-2 (dilution, 1:200 in PBS containing 5\% BSA). Following three washes with PBS, a biotin-labelled secondary antibody (dilution, 1:1,000 in PBS containing 5\% BSA) was applied for $30 \mathrm{~min}$, prior to the addition of streptavidin, in order to form the streptavidin-biotin complex. Next, DAB was used to visualize the final products of the immunoreaction. Immunoreactivity for VEGF and FGF-2 was counted in five randomly selected fields. The results were classified as negative ( () , weakly positive $\left(^{+}\right)$, positive $\left({ }^{++}\right)$, and strongly positive $\left({ }^{(++}\right)$, depending on the intensity of the signal.

Statistical analysis. The results were expressed as the mean \pm standard deviation of three independent experiments. Data were analyzed by comparison with the control group via the Student's t-test. $\mathrm{P}<0.05$ was considered to indicate a statistically significant difference.

\section{Results}

Inhibition of HUVEC proliferation by CPU-XT-008. The HUVEC line is frequently used as a model to study the function of endothelial cells in tumor angiogenesis. To quantify HUVEC proliferation, an MTT assay was performed. As depicted in Fig. 1, once the NBCS-induced HUVEC proliferation model had been established, the cells were treated with different concentrations of CPU-XT-008 for $24 \mathrm{~h}$, prior to be subjected to MTT assay. The proliferative activity
A
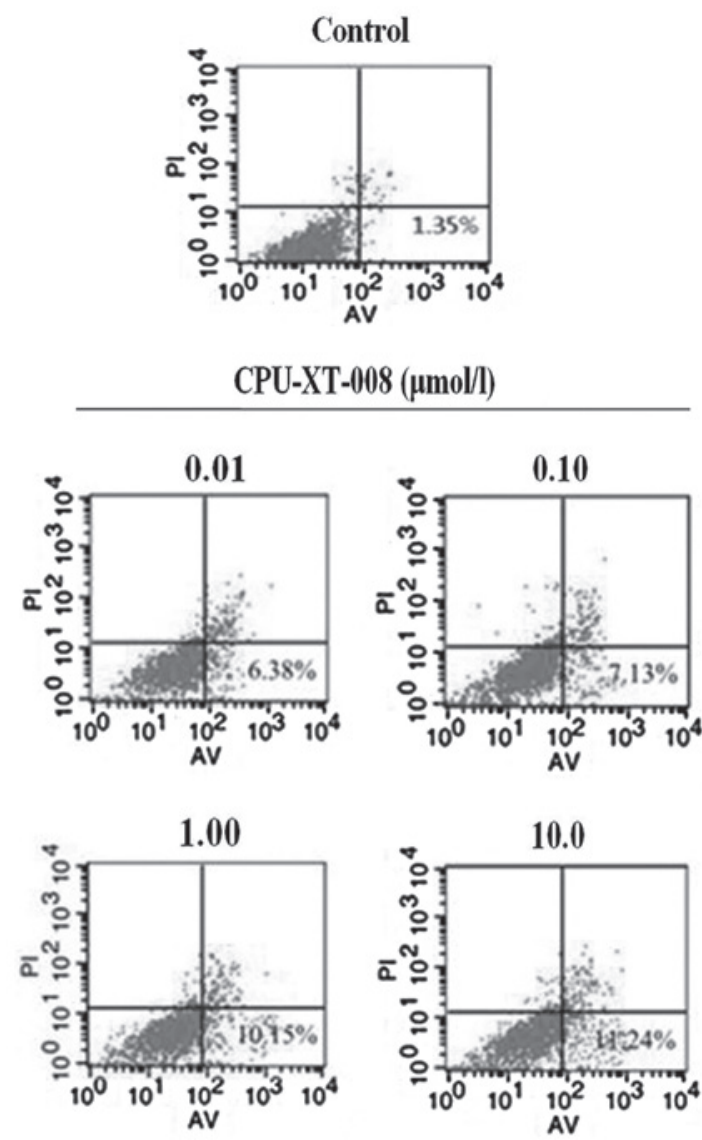

B

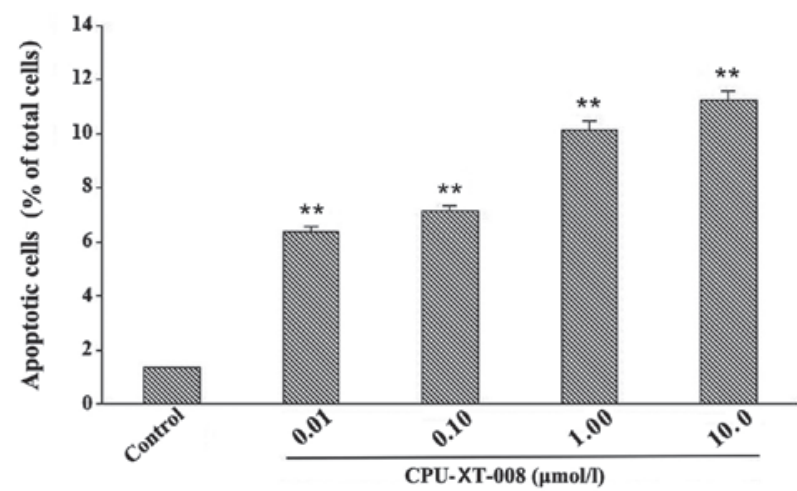

Figure 2. CPU-XT-008 induces the apoptosis of human umbilical vein endothelial cells in a dose-dependent manner. (A) Apoptosis was measured by AV/PI staining in flow cytometry. Control cells were treated with DMEM + 10\% NBCS, while test groups were treated with DMEM + 10\% NBCS + CPU-XT-008 (0.01, 0.10, 1.00 and $10.0 \mu \mathrm{mol} / 1$, respectively). (B) Compared with the control group, the percentage of apoptotic cells increased upon treatment with CPU-XT-008. Values are expressed as the mean \pm standard deviation, $\mathrm{n}=3$. $^{* *} \mathrm{P}<0.01$ vs. Control group. $\mathrm{AV}$, annexin $\mathrm{V}$; PI, propidium iodide; DMEM, Dulbecco's modified Eagle's medium; NBCS, new born calf serum.

of HUVECs was significantly reduced by CPU-XT-008, compared with the control group. Cells exposed to $0.01,0.10,1.00$ and $10.0 \mu \mathrm{mol} / \mathrm{l}$ CPU-XT-008 exhibited a percentage of inhibition of 35.5, 37.1, 49.2 and $54.8 \%$, respectively, in their proliferative ability. Thus, CPU-XT-008 inhibited the proliferation of HUVECs in a dose-dependent manner. 
A

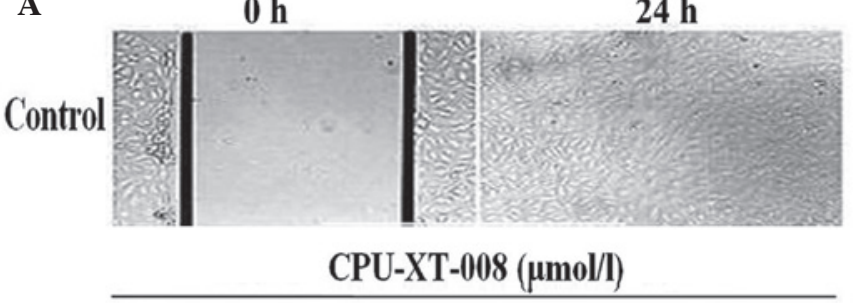

0.01

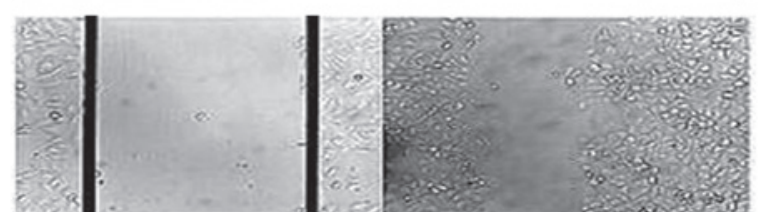

0.10

1.00

10.0
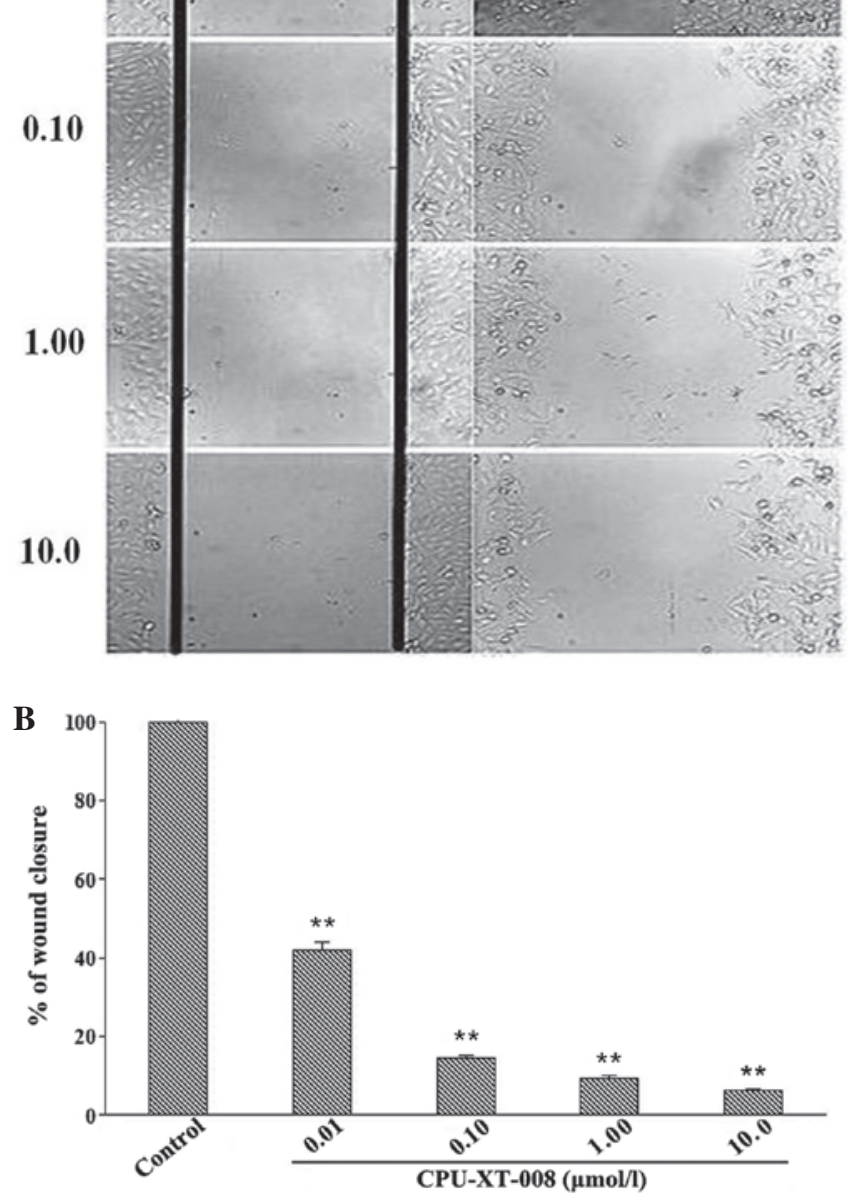

Figure 3. CPU-XT-008 inhibits HUVEC migration. (A) Confluent HUVEC monolayers were scratch-wounded, treated with different concentrations of CPU-XT-008, and photographed $24 \mathrm{~h}$ later at $\mathrm{x} 40$ magnification (bar $=10 \mu \mathrm{m})$. The black lines in the image indicate the denuded areas. Control cells were treated with DMEM $+10 \%$ NBCS, whereas test groups were treated with DMEM +10\% NBCS + CPU-XT-008 $(0.01,0.10,1.00$ and $10.0 \mu \mathrm{mol} / 1$, respectively). (B) Cell migration was quantified as the percentage of wound closure between 0 and $24 \mathrm{~h}$. Values are expressed as the mean \pm standard deviation, $n=3$. ${ }^{* *} \mathrm{P}<0.01$ vs. Control group. HUVEC, human umbilical vein endothelial cell; DMEM, Dulbecco's modified Eagle's medium; NBCS, new born calf serum.

CPU-XT-008 induced HUVEC apoptosis. To elucidate the mechanism by which CPU-XT-008 exerted its anti-proliferative activity on HUVECs, the effect of CPU-XT-008 on inducing endothelial cell apoptosis was investigated by flow cytometry. The results of the experiment are presented in Fig. 2. The percentage of cells in the early stages of apoptosis in the control group was $1.35 \pm 0.25 \%$. Following treatment with

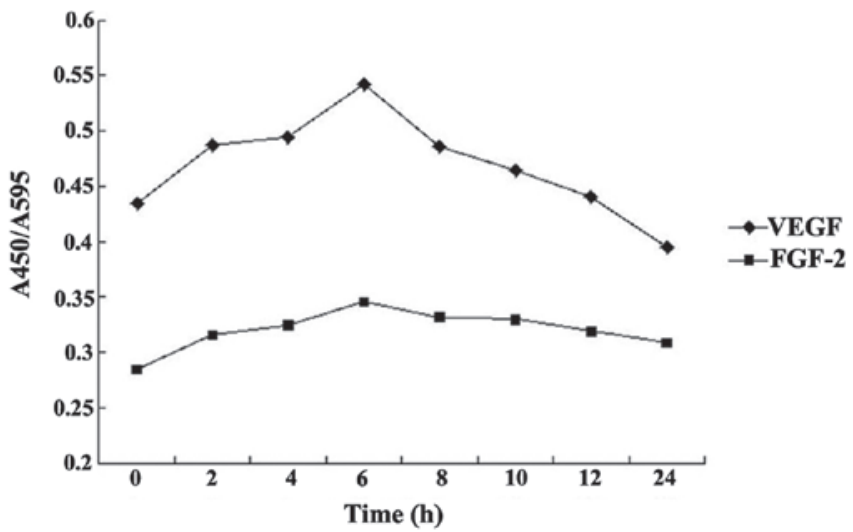

Figure 4. Determination of the induction time for maximal expression of VEGF and FGF-2 by NBCS. Human umbilical vein endothelial cells were exposed to $10 \%$ NBCS for $0,2,4,6,8,10,12$ and $24 \mathrm{~h}$, and the protein expression levels of VEGF and FGF-2 were determined by enzyme-linked immunosorbent assay. VEGF, vascular endothelial growth factor; FGF-2, basic fibroblast growth factor; NBCS, new born calf serum; A, absorbance.

A
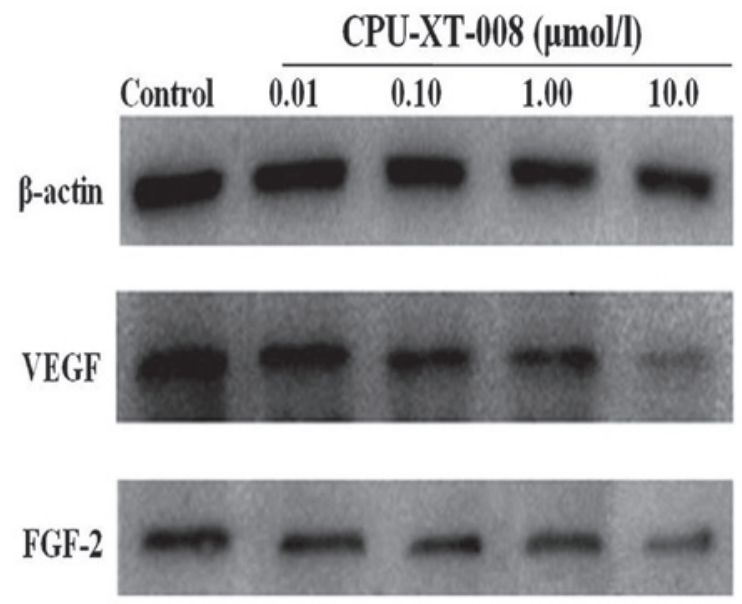

B

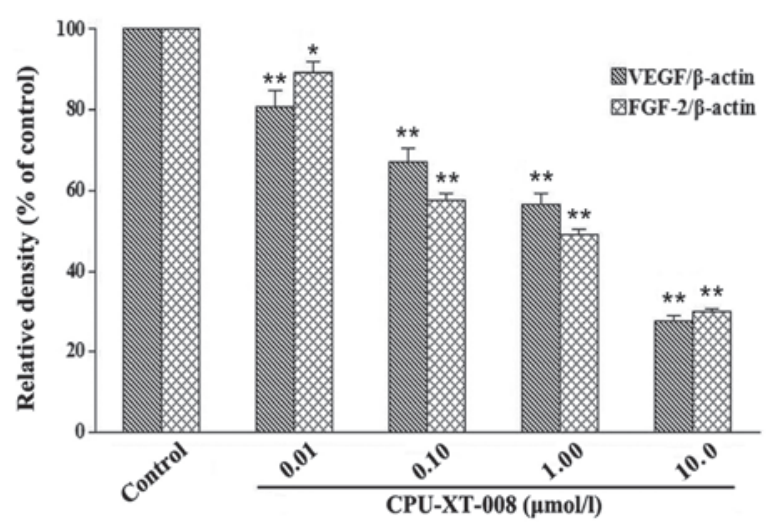

Figure 5. Western blot analysis of the effect of CPU-XT-008 on the protein expression levels of VEGF and FGF-2 in HUVECs, following 24-h exposure to $0.01,0.10,1.00$ and $10.0 \mu \mathrm{mol} / 1 \mathrm{CPU}-\mathrm{XT}-008$, respectively. (A) Representative protein levels of VEGF, FGF-2 and $\beta$-actin in CPU-XT-008-treated HUVECs, as determined by western blotting. (B) Optical density analysis representing the ratio of the protein levels of VEGF and FGF- 2 to those of $\beta$-actin, , which was used as internal control. Values are expressed as the mean \pm standard deviation, $\mathrm{n}=3$. ${ }^{*} \mathrm{P}<0.05 ;{ }^{* * *} \mathrm{P}<0.01$ vs. Control group. HUVEC, human umbilical vein endothelial cells; VEGF, vascular endothelial growth factor; FGF-2, basic fibroblast growth factor. 
A

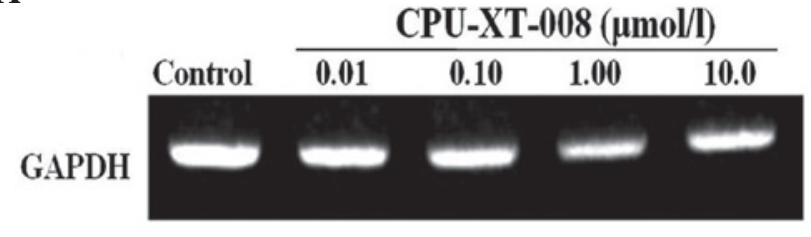

VEGF

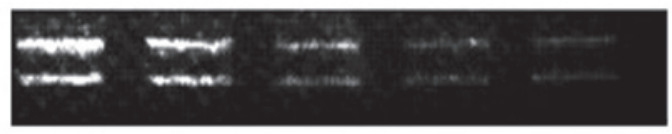

FGF-2

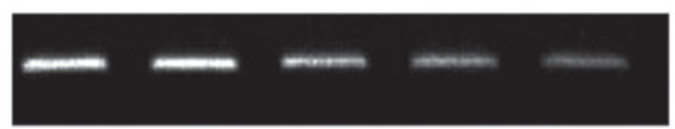

B

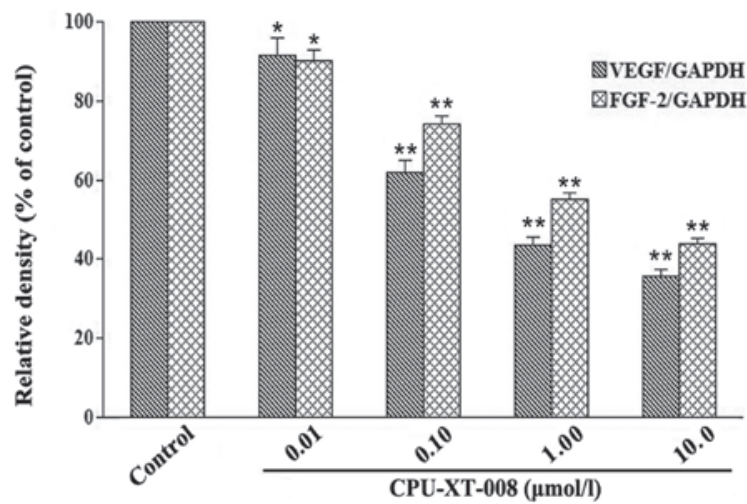

Figure 6. Reverse transcription-PCR analysis of the effect of CPU-XT-008 (0.01, 0.10, 1.00 and $10.0 \mu$ mol/1, respectively) on the mRNA expression levels of on the mRNA expression levels of two different isoforms of VEGF-A (VEGF165 and VEGF121) and FGF-2 on human umbilical vein endothelial cells. (A) Agarose gel electrophoresis of the PCR products corresponding to the amplification of VEGF and FGF-2. (B) Optical density analysis representing the percentage of VEGF-A and FGF-2 mRNA vs. GAPDH mRNA, which was used as control. Values are expressed as the mean \pm standard deviation, $\mathrm{n}=3$. * $<0.05$; ${ }^{* *} \mathrm{P}<0.01$ vs. control group. PCR, polymerase chain reaction; VEGF, vascular endothelial growth factor; FGF-2, basic fibroblast growth factor. mRNA, messenger RNA; GAPDH, glyceraldehyde 3-phosphate dehydrogenase.

CPU-XT-008 at $0.01,0.10,1.00$ and $10.0 \mu \mathrm{mol} / 1$ for $24 \mathrm{~h}$, the percentage of cells in the early stages of apoptosis increased to $6.38 \pm 0.33,7.13 \pm 0.45,10.15 \pm 0.22$ and $11.24 \pm 0.55 \%$, respectively. Thus, CPU-XT-008 induced apoptosis in HUVECs in a dose-dependent manner.

CPU-XT-008 inhibited HUVEC migration. The anti-migration properties of CPU-XT-008 were studied in a wound-healing assay using HUVECs. The size of the wound was inspected microscopically over time to monitor the healing of the wound by the migration of the cells. As observed in Fig. 3A, the wound completely healed within $24 \mathrm{~h}$ in the control group, due to cell migration, whereas this process was inhibited in the group treated with CPU-XT-008. Fig. 3B represents the percentage of inhibition of cell migration exhibited by HUVECs $(42.08$, $14.59,9.58$ and $6.25 \%$ ) upon 24 -h treatment with $0.01,0.10$, 1.00 and $10.0 \mu \mathrm{mol} / 1 \mathrm{CPU}-\mathrm{XT}-008$, respectively. These results demonstrate that CPU-XT-008 inhibited HUVEC migration in a dose-dependent manner, similarly to the effect exerted by this compound on HUVEC proliferation.

Determination of time for maximal NCBS-induced expression of VEGF and FGF-2. A cell-based ELISA was used to determine the time at which the NCBS-induced expression levels of VEGF and FGF-2 were maximum. As presented in Fig. 4, 10\% NBCS induced expression of VEGF and FGF-2 in HUVECs upon $0,2,4,6,8,10,12$ and 24 -h incubation. The protein expression levels of VEGF and FGF-2 increased following exposure to NBCS in a time-dependent manner, reaching their maximal values at $6-\mathrm{h}$. At this point, the maximum protein expression levels of VEGF and FGF-2 were 1.25 and 1.21-fold higher, respectively, in NBCS-stimulated HUVECs than in non-NBCS-stimulated cells. The protein expression levels of VEGF and FGF-2 gradually reduced to the initial levels with increasing times of exposure to NBCS. The incubation times at which these proteins displayed their maximum expression levels were used as induction times in the subsequent western blotting and immunocytochemistry experiments.
CPU-XT-008 reduced the protein expression levels of VEGF and FGF-2 in HUVECs. To investigate whether CPU-XT-008 was able to inhibit the protein expression of VEGF and FGF-2 in HUVECs, the cells were incubated for $24 \mathrm{~h}$ with increasing concentrations of CPU-XT-008, and the protein expression levels of VEGF and FGF-2 were determined by western blotting. The results are presented in Fig. 5 . The protein expression levels of VEGF and FGF-2 in the control group were equated to $100 \%$, and used for normalization of the results. The treatment with CPU-XT-008 inhibited the protein expression of VEGF and FGF-2 in HUVECs. The percentage of inhibition for VEGF and FGF-2 was 19.1, 33.0, 43.6 and $72.3 \%$, and $10.6,42.6,51.1$ and $70.2 \%$, respectively, when the cells were subjected to $0.01,0.10,1.00$ and $10.00 \mu \mathrm{mol} / 1$ CPU-XT-008.

CPU-XT-008 reduced the $m R N A$ expression levels of VEGF and FGF-2 in HUVECs. To determine whether CPU-XT-008 was able to regulate protein production at the transcriptional level, the expressions of two different isoforms of VEGF-A (VEGF165 and VEGF121) and FGF-2 were analyzed by RT-PCR. The results obtained were normalized by equating the mRNA levels measured for the control group to $100 \%$. As presented in Fig. 6, the treatment of HUVECs with CPU-XT-008 reduced the mRNA expression levels of both VEGF-A isoforms and FGF-2, compared with the untreated control group. Upon exposure of HUVECs to $0.01,0.10,1.00$ and $10.0 \mu \mathrm{mol} / 1 \mathrm{CPU}-\mathrm{XT}-008$, the percentage of inhibition of mRNA expression was 8.5, 38.1, 56.5 and $64.4 \%$ for VEGF, and 9.6, 25.9, 45.0 and $56.1 \%$ for FGF-2, respectively.

Immunocytochemistry analysis of VEGF and FGF-2 expression in HUVECs. Immunocytochemistry was used to localize the intracellular expression of VEGF and FGF-2 in HUVECs subjected to treatment with CPU-XT-008. The protein expression levels of VEGF and FGF-2 are presented in Fig. 7A and B, respectively. Positive signal was defined as the presence of brown-yellow particles in the cytoplasm. The 
A

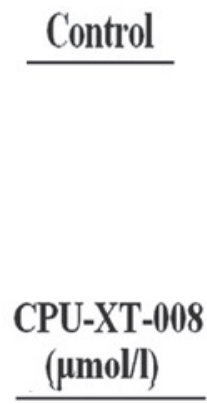

0.01

0.10

1.00

10.0

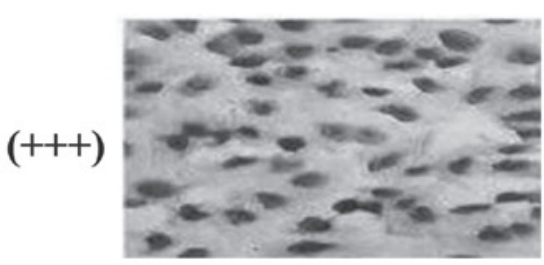

B
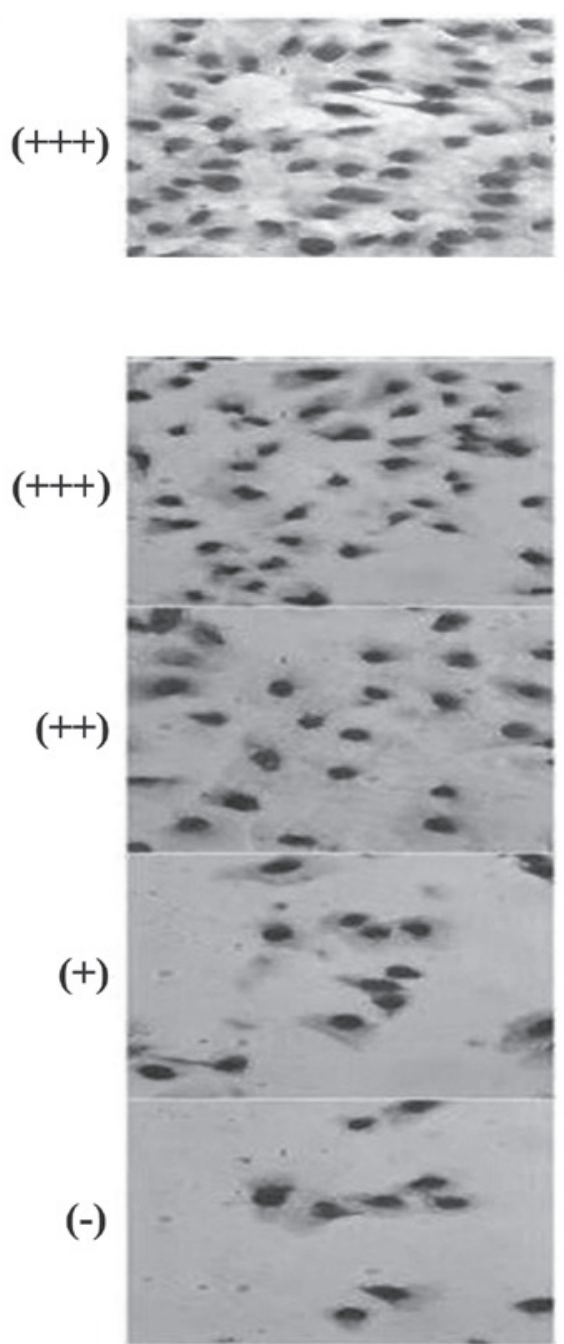

Figure 7. Immunocytochemistry analysis of the effect of CPU-XT-008 on the protein expression levels of (A) VEGF and (B) FGF-2 in human umbilical vein endothelial cells. The cells in the control group were treated with DMEM + 10\% NBCS, whereas the cells in the test groups were exposed to DMEM + 10\% NBCS + CPU-XT-008 (0.01, 0.10, 1.00 and $10.0 \mu \mathrm{mol} / 1$, respectively). Immunoreactivity for VEGF and FGF-2 was counted in five randomly selected fields. The results were classified as negative $\left({ }^{-}\right)$, weakly positive $\left({ }^{+}\right)$, positive $\left({ }^{++}\right)$and strongly positive $\left(^{+++}\right)$, depending on the intensity of the signal. VEGF, vascular endothelial growth factor; FGF-2, basic fibroblast growth factor; DMEM, Dulbecco's modified Eagle's medium; NBCS, new born calf serum.

cytoplasm of the cells in the control groups was clearly stained brown-yellow, implying that the expression of VEGF and FGF-2 was strongly positive in these cells $\left({ }^{++}\right)$. In HUVECs treated with CPU-XT-008 at 0.01, 0.10, 1.00 and $10.0 \mu \mathrm{mol} / \mathrm{l}$, the signals for VEGF were described as strongly positive $\left({ }^{+++}\right)$, positive $\left({ }^{++}\right)$, weakly positive $\left(^{+}\right)$and weakly positive $\left(^{+}\right.$), respectively, compared with the control group. For FGF-2, the signals were observed to be strongly positive $\left(^{+++}\right)$, positive $\left({ }^{++}\right)$, weakly positive $\left(^{+}\right)$and negative $\left(^{-}\right)$, respectively, compared with the control group. These results suggested that the protein expression of VEGF and FGF-2 in HUVECs was inhibited by CPU-XT-008 in a dose-dependent manner.

\section{Discussion}

Due to the solubility and bioavailability limitations of CA-4, research on CA-4 derivatives for cancer anti-angiogenic therapy has become a topic of interest in recent years (15).
However, a limited numbers of CA-4 derivatives, including the water-soluble prodrug CA-4 disodium salt and AVE8062 (Sanofi S.A., Paris, France), have progressed through to phase III clinical trials (16). The latter compound has been reported to currently be in phase III evaluation (clinical trial no. NCT00699517) for the treatment of advanced-stage soft tissue sarcoma, following the failure of anthracycline and ifosfamide chemotherapies (17).

Heparin is an endogenous multi-target macromolecule capable of regulating the activity of angiogenic factors (18). The amino sugar-containing main fragment of heparin simulates the heparin molecular structure, and is expected to inhibit the growth of blood vessels (19). Therefore, the Medicinal Chemistry Department of China Pharmaceutical University previously designed and synthesized a series of small molecule N-glycosylation amide class CA-4 analogues, from which CPU-XT-008 was identified as the most active compound. 
Endothelial cells present in tumors participate in the formation of blood vessels, and the 'switch' to an angiogenic phenotype is a hallmark of tumor malignancy (20). The angiogenic process involves multiple steps, including matrix degradation, migration, proliferation and differentiation into a tube-like structure (21). The present study has demonstrated that CPU-XT-008 significantly inhibits the proliferation of HUVECs in a dose-dependent manner. In addition, the mechanism by which this compound induced the death of proliferating endothelial cells was also investigated in the present study. The results indicated that $0.01 \mu \mathrm{mol} / 1$ CPU-XT-008 significantly stimulated HUVEC apoptosis. Therefore, CPU-XT-008 may be an important target for cancer anti-vascular therapy. Previous studies have suggested that the cytotoxicity induced by CA-4 is due to its ability to bind to the colchicine-binding site on tubulin (22). Furthermore, previous studies have reported that CA-4 inhibits the polymerization of tubulin, arrests cancer cells in the $\mathrm{G}_{2} / \mathrm{M}$ phase of the cell cycle and induces caspase-dependent apoptosis in human leukaemia cells (23). Thus, CA-4 exhibits promising anticancer therapeutic potential.

Concomitant with the inhibition of cell proliferation, a significant inhibitory effect of CPU-XT-008 on HUVEC migration was demonstrated in the wound-healing assay conducted in the present study. The results indicated that CPU-XT-008 inhibited proliferation and migration, and induced apoptosis in endothelial cells. Therapeutically, these mechanisms must be overcome, since they are considered to be involved in the prevention of tumor re-growth during the chronic dosing schedules employed in clinical trials (24).

To explore a possible mechanism for the anti-angiogenic properties of CPU-XT-008, the effects of this compound on the protein expression levels of VEGF and FGF-2 in HUVECs were investigated in the present study. The results suggested that CPU-XT-008 may indirectly influence angiogenesis, since $0.01 \mu \mathrm{mol} / 1 \mathrm{CPU}-\mathrm{XT}-008$ was observed to significantly reduce the protein expression levels of VEGF and FGF-2 by 19.1 and $10.6 \%$, respectively. In addition to its angiogenic activity, VEGF is also able to protect endothelial cells from apoptosis by stimulating the activation of survival pathways such as the phosphoinositol-3-kinase signaling pathway, and the upregulation of anti-apoptotic proteins, including B-cell lymphoma 2 and, in particular, survivin, which is an important microtubule-binding agent that inhibits apoptosis and regulates the mitotic spindle in endothelial cells $(25,26)$. FGF- 2 is a basic protein that promotes mitosis and angiogenesis (27). When FGF-2 binds to specific receptors, cell metabolism is enhanced by the activation of intracellular tyrosine kinases in the target cell (28). Additionally, FGF-2 is also involved in the formation of tumor blood vessels (27). In the present study, CPU-XT-008 inhibited the mRNA and protein expression of VEGF and FGF-2, suggesting that CPU-XT-008 controls the production of VEGF and FGF-2 at the transcriptional level.

In conclusion, the results of the present study revealed that CPU-XT-008 efficiently inhibited proliferation and migration, and induced apoptosis of HUVECs, in a dose-dependent manner. In addition, CPU-XT-008 also reduced the protein expression levels of VEGF and FGF-2, potentially by reducing their mRNA levels at the transcriptional stage. Overall, these results suggest that CPU-XT-008 exhibits similar efficiency to
CA-4 as a novel vascular targeting agent. Furthermore, due to the biocompatibility and molecular targeting properties exhibited by CPU-XT-008, further studies on this compound for the development of antitumor drugs are worthwhile.

\section{Acknowledgements}

The present study was supported by the Priority Academic Program Development of Jiangsu Higher Education Institution (Nanjing, China) (grant no. CXLX11 0801). The authors would like to thank the Biochemistry and Microbiology Laboratory of China Pharmaceutical University (Nanjing, China) for providing HUVECs.

\section{References}

1. Kerbel RS: Tumor angiogenesis. N Engl J Med 358: 2039-2049, 2008.

2. Roodink I and Leenders WP: Targeted therapies of cancer: Angiogenesis inhibition seems not enough. Cancer Lett 299: 1-10, 2010.

3. Park HE, Baek SH, Min J, Chang YT, Kang DK, Chang SI and Joe YA: Myoseverin is a potential angiogenesis inhibitor by inhibiting endothelial cell function and endothelial progenitor cell differentiation. DNA Cell Biol 25: 514-522, 2006.

4. Kretzschmann VK and Furst R: Plant-derived vascular disrupting agents: Compounds, actions, and clinical trials. Phytochem Rev 13: 191-206, 2014.

5. Hanahan D and Folkman J: Patterns and emerging mechanisms of the angiogenic switch during tumorigenesis. Cell 86: 353-364, 1996.

6. Van Beijnum JR, Dings RP, van der Linden E, Zwaans BMM, Ramaekers FCS, Mayo KH and Griffioen AW: Gene expression of tumor angiogenesis dissected: Specific targeting of colon cancer angiogenic vasculature. Blood 108: 2339-2348, 2006.

7. Ghilardi C, Chiorino G, Dossi R, Nagy Z, Giavazzi R and Bani MR: Identification of novel vascular markers through gene expression profiling of tumor-derived endothelium. BMC Genomics 9: 201, 2008.

8. Torijano-Gutiérrez S, Díaz-Oltra S, Falomir E, Murga J, Carda M and Marco JA: Synthesis of combretastatin A-4 O-alkyl derivatives and evaluation of their cytotoxic, antiangiogenic and antitelomerase activity. Bioorg Med Chem 21: 7263-7274, 2013.

9. Cross MJ and Claesson-Welsh L: FGF and VEGF function in angiogenesis: Signalling pathways, biological responses and therapeutic inhibition. Trends Pharmacol Sci 22: 201-207, 2001.

10. Casanovas O, Hicklin DJ, Bergers G and Hanahan D: Drug resistance by evasion of antiangiogenic targeting of VEGF signaling in late-stage pancreatic islet tumors. Cancer Cell 8: 299-309, 2005.

11. Aalinkeel R, Nair MP, Sufrin G, Mahajan SD, Chadha KC, Chawda RP and Schwartz SA: Gene expression of angiogenic factor correlates with metastatic potential of prostate cancer cells. Cancer Res 64: 5311-5321, 2004.

12. Pettit GR, Singh SB, Hamel E, Lin CM and Alberts DS, Garcia-Kendall D: Isolation and structure of the strong cell growth and tubulin inhibitor combretastatin A-4. Experientia 45: 209-211, 1989.

13. Krilleke D, DeErkenez A, Schubert W, Giri I, Robinson GS, $\mathrm{Ng}$ YS and Shima DT: Molecular mapping and functional characterization of the VEGF164 heparin-binding domain. J Biol Chem 282: 28045-28056, 2007.

14. Parihar S, Kumar A, Chaturvedi AK, Sachan NK, Luqman S, Changkija B, Manohar M, Prakash O, Chanda D, Khan F, et al: Synthesis of combretastatin A4 analogues on steroidal framework and their anti-breast cancer activity. J Steroid Biochem Mol Biol 137: 332-344, 2013

15. Böhle AS, Leuschner I, Kalthoff H and Henne-Bruns D: Combretastatin A-4 prodrug: A potent inhibitor of malignant hemangioendothelioma cell proliferation. Int J Cancer 87: 838-843, 2000.

16. Petit I, Karajannis MA, Vincent L, Young L, Butler J, Hooper AT, Shido K, Steller H, Chaplin DJ, Feldman E and Rafii S: The microtubule-targeting agent $\mathrm{CA} 4 \mathrm{P}$ regresses leukemic xenografts by disrupting interaction with vascular cells and mitochondrial-dependent cell death. Blood 111: 1951-1961, 2008. 
17. Kremmidiotis G, Leske AF, Lavranos TC, Beaumont D, Gasic J, Hall A, O'Callaghan M, Matthews CA and Flynn B: BNC105: A novel tubulin polymerization inhibitor that selectively disrupts tumor vasculature and displays single-agent antitumor efficacy. Mol Cancer Ther 9: 1562-1573, 2010.

18. Khachigian LM and Parish CR: Phosphomannopentaose sulfate (PI-88): heparan sulfate mimetic with clinical potential in multiple vascular pathologies. Cardiovase Drug Rev 22: 1-6, 2004.

19. Norrby K: Low-molecular-weight heparins and angiogenesis. APMIS 114: 79-102, 2006.

20. Bussolati B, Deregibus MC and Camussi G: Characterization of molecular and functional alterations of tumor endothelial cells to design anti-angiogenic strategies. Curr Vasc Pharmacol 8: 220-232, 2010.

21. Zhou J, Jin J, Zhang Y, Yin Y, Chen X and Xu B: Synthesis and antiproliferative evaluation of novel benzoimidazole-contained oxazole-bridged analogs of combretastatin A-4. Eur J Med Chem 68: 222-232, 2013.

22. Dark GG, Hill SA, Prise VE, Tozer GM, Pettit GR and Chaplin DJ: Combretastatin A-4, an agent that displays potent and selective toxicity toward tumor vasculature. Cancer Res 57: 1829-1834, 1997

23. Magalhães HI, Wilke DV, Bezerra DP, Cavalcanti BC, Rotta R, de Lima DP, Beatriz A, Moraes MO, Diniz-Filho J and Pessoa C: (4-Methoxyphenyl)(3,4,5-trimethoxyphenyl) methanone inhibits tubulin polymerization, induces $\mathrm{G}_{2} / \mathrm{M}$ arrest, and triggers apoptosis in human leukemia HL-60 cells. Toxicol App Pharmacol 272: 117-126, 2013.
24. Nathwani SM, Hughes L, Greene LM, Carr M, O'Boyle NM, McDonnell S, Meegan MJ and Zisterer DM: Novel cis-restricted $\beta$-lactam combretastatin A-4 analogues display anti-vascular and anti-metastatic properties in vitro. Oncol Rep 29: 585-594, 2013.

25. Nör JE, Christensen J, Liu J, Peters M, Mooney DJ, Strieter RM and Polverini PJ: Up-regulation of Bcl-2 in microvascular endothelial cells enhances intratumoral angiogenesis and accelerates tumor growth. Cancer Res 61: 2183-2188, 2001.

26. Tran J, Master Z, Yu JL, Rak J, Dumont DJ and Kerbel RS: A role for survivin in chemoresistance of endothelial cells mediated by VEGF. Proc Natl Acad Sci 99: 4349-4354, 2002.

27. Xu CJ, Mikami T, Nakamura T, Tsuruta T, Nakada N, Yanagisawa N, Jiang SX and Okayasu I: Tumor budding, myofibroblast proliferation and fibrosis in obstructing colon carcinoma: The roles of Hsp47 and basic fibroblast growth factor. Pathol Res Pract 209: 69-74, 2013.

28. Plum SM, Holaday JW, Ruiz A, Madsen JW, Fogler WE and Fortier AH: Administration of a liposomal FGF-2 peptide vaccine leads to abrogation of FGF-2-mediated angiogenesis and tumor development. Vaccine 19: 1294-1303, 2001. 